\title{
Tradução e Adaptação da Escala CARSAL/CARVAL para Portugal: Estudo psicométrico
}

\author{
José Mendes ${ }^{1,2}$, Rui Rego ${ }^{3,4}$ \& Vera Pereira ${ }^{3,4}$ \\ ${ }^{1}$ INTELECTO - Psicologia \& Investigação, Ponta Delgada, Portugal \\ ${ }^{2}$ Instituto de Psicologia Cognitiva e Desenvolvimento Humano e Social (IPCDHS-UC), Coimbra, Portugal \\ ${ }^{3}$ Research Center for Spatial and Organizational Dynamics, Faro, Portugal \\ ${ }^{4}$ UALG - Faculty of Human and Social Sciences, University of Algarve, Campus of Gambelas, Faro, Portugal
}

\begin{abstract}
Resumo: 0 presente estudo tem como objetivo a tradução, adaptação e análise das propriedades psicométricas do instrumento CARSAL/CARVAL. A amostra foi constituída por 202 adultos, com idades entre os 18 e os 68 anos, oriundos de várias regiões de Portugal Continental e Ilhas. Avaliaram-se neste estudo, questões sociodemográficas; dois aspetos do auto-esquema da aparência; o investimento esquemático, a autoconsciência da aparência; e os afetos positivos e afetos negativos. A análise fatorial exploratória permitiu a identificação da estrutura fatorial subjacente, confirmando os itens de cada dimensão. Os resultados sugerem índices de consistência interna adequados em ambas as dimensões, verificando-se a homogeneidade das variáveis. A análise fatorial confirmatória apresentou bom ajustamento, apontando para um modelo ajustado a dois fatores, composto por 13 itens. A CARSAL/CARVAL, apresenta-se como um instrumento psicometricamente robusto, na avaliação dos dois aspetos do auto-esquema da aparência (saliência/valência).
\end{abstract}

Palavras-chave: Aparência; auto-esquema; saliência; valência; análise fatorial.

Translation and Adaptation of the CARSAL / CARVAL Scale to Portugal: a psychometric study: This research aims to study the translation, adaptation and psychometric properties analyze of the CARSAL / CARVAL instrument. The sample consisted of 202 adults, aged between 18 and 68 years old, from various regions of mainland Portugal and its islands. The participants answered sociodemographic questions; two aspects of appearance self-schema; schematic investment and appearance self-awareness; and positive and negative affect. The exploratory factor analysis allowed the underlying factor structure identification, confirming the items of each dimension. The results suggested adequate internal consistency indices in both dimensions, verifying the variables homogeneity. Confirmatory factorial analysis presented good adjustment, indicating a two-factor adjusted model, composed of 13 items. The CARSAL / CARVAL as a psychometrically robust instrument in the evaluation of the two aspects of appearance self-schema (salience / valence).

Keywords: Appearance; self-schema; salience; valence; factorial analysis.

Mendes (2017) evidenciou a existência de uma relação entre as características da personalidade, investimento esquemático e autoconsciência da aparência, que influencia o ajustamento psicológico do indivíduo às diferenças visíveis (desfiguramento). Desde a infância, que o desenvolvimento cognitivo influencia na construção da imagem corporal (Hockenberry \& Wilson, 2011), através de dois atributos centrais (investimento e avaliação), auxiliados pelo processamento de esquemas da aparência (Cash \& Pruzinsky, 2004; Cash \& Smolak, 2012).

A imagem corporal é um constructo multidimensional (Cash, 2006), exigindo-se assim uma distinção entre o conceito de esquema corporal e imagem corporal. Pitron e De Vignemont (2017) defendem o envolvimento do esquema corporal na ação, enquanto que a imagem corporal é a perceção que o individuo tem do seu corpo. A distinção entre estes conceitos (ação e perceção) requerem diferentes transformações (sensoriais, corticais e subcorticais) e permanecem inerentemente distintas em relação à sua função (Pitron \& De Vignemont, 2017). No entanto, quer o esquema corporal quer a imagem corporal de longo prazo podem apresentar os mesmos tipos de distorção corporal (Pitron, Alsmith, \& De Vignemont, 2018).

Nazaré, Moreira e Canavarro (2010) referem que o investimento na aparência se encontra intrinsecamente ligado ao auto-esquemas através da saliência motivacional (grau de atenção na gestão da

${ }^{1}$ Morada para correspondência: José Mendes, Rua Duarte Pimentel, 52B, Fração L, R/C Esq., 9500-451 Ponta Delgada, Açores, Portugal. Email: josemendes@intelecto.pt 
aparência) e à saliência auto-avaliativa (integração da identidade do indivíduo permitindo o seu valor pessoal). Mendes e Pereira (2018), por sua vez, mencionam que a avaliação da imagem corporal se concentra na autoconsciência da aparência, isto é, o indivíduo foca os sentimentos negativos e comportamentos relativamente a algum aspeto que o incomoda na sua aparência.

Moss e Rosser (2012) referem que os esquemas da aparência se concetualizam como uma representação cognitiva da informação organizada sobre o self em relação à aparência, incluindo emoções sobre a mesma. Estes esquemas cognitivos contêm crenças e suposições condicionais com impacto no self (Clarke, Thompson, Jenkinson, Rumsey, \& Newell, 2014), considerando-se um aspeto importante na construção dos esquemas da aparência (Deno et. al., 2012). Cash (2006) defende os auto-esquemas da aparência como parte integrante do autoconceito, em que uma alteração na aparência pode influenciar uma nova construção de esquemas da aparência, tornando-se mais evidente a presença de erros cognitivos e emoções negativas (Brill, Clarke, Veale, \& Butler, 2006).

Apesar de estudos verificarem que os processos emocionais em relação à aparência também desempenham um papel importante no ajustamento psicológico às diferenças visíveis (Mendes, 2017; Moss, Lawson, White, \& The Appearance Research Collaboration, 2014), os fatores que promovem/mantêm a insatisfação com a imagem corporal ainda permanecem obscuros (Lonergan et al., 2019). Um estudo de Fuller-Tyszkiewicz et al., (2019) revelou que as comparações e os comentários (positivos e negativos) relacionados com a aparência são preditores de mudanças na insatisfação com o corpo. Assim revela-se pertinente compreender os esquemas corporais, dado que estes têm alguma primazia sobre a imagem corporal (Pitron et al., 2018).

0 conjunto de itens que constituem a CARSAL/CARVAL, surgem da união da escala Center for Appearance Research Salience (CARSAL) composta por 10 itens e a escala Center for Appearance Research Valence (CARVAL) composta por 12 itens. Estas escalas foram geradas a partir da experiência clínica dos autores e desenvolvidas de acordo com base numa cuidadosa operacionalização teórica dos constructos: saliência e valência. Após o estudo e análise das propriedades psicométricas da união das duas escalas, estas ficaram reduzidas a uma escala de 13 itens, revelando-se robusta quer para a população geral (Moss \& Rosser, 2012) quer para a população clínica (Moss et al., 2014).

A CARSAL/CARVAL, foi desenvolvida com a intenção de avaliar dois aspetos esquemáticos da aparência: até que ponto a aparência e o eu físico são trazidos à perceção consciência (saliência) e até que ponto o individuo avalia sua aparência de maneira positiva/negativa (valência). Esta medida breve, válida e confiável da saliência e valência da aparência, avalia a contribuição preditiva independente e moderada desses fatores em relação às dificuldades psicológicas inerentes à autoconsciência da aparência (Moss \& Rosser, 2012; Moss et al., 2014).

A comunidade científica em Portugal tem-se debruçado sobre as preocupações com a aparência (Mendes \& Pereira, 2018). Recentemente, um estudo longitudinal reforçou a necessidade de escalas breves, que avaliem as preocupações com a aparência, em contexto clínico (Mendes, 2017), para uma melhor compreensão dos paradigmas associados às preocupações com a aparência e/ou esquemas de aparência. Neste seguimento, torna-se ainda pertinente referir que vários estudos levados a cabo têm defendido a necessidade da construção de escalas que visem a avaliação das preocupações com a aparência, com o objetivo de compreender as características individuais no ajustamento psicológico às diferenças visíveis (Carr, Moss, \& Harris, 2005; Lansdown, Rumsey, Bradbury, Carr, \& Partridge, 1997; Mendes, Figueiras, Moreira, \& Moss, 2016).

Pretende-se com a CARSAL/CARVAL contribuir para a avaliação dos componentes distintivos do autoconceito relacionado com os auto-esquemas da aparência, diferenciando os componentes organizacionais (saliência) e avaliativos (valência), e contribuir para uma melhor avaliação da autoconsciência da aparência. 0 objetivo deste estudo é traduzir e adaptar a CARSAL/CARVAL para a população portuguesa, apresentando o respetivo estudo psicométrico, respeitando as instruções definidas por vários autores, no desenvolvimento de instrumentos de avaliação (Smith, McCarthy, \& Anderson, 2000; Tambs \& Røysamb, 2014; Widaman, Little, Preacher, \& Sawalani, 2011).

\section{MÉTODO}

\section{Participantes}

A amostra do estudo é aleatória simples e foi constituída por 202 participantes de ambos os sexos, com idades compreendidas entre os 18 e os 68 anos $(M=30.35, D P=10.73)$, que estudaram uma média de 14.98 anos $(D P=3.73)$. A maioria dos participantes pertence ao género feminino $(164 ; 81.2 \%)$, em detrimento dos do género masculino (38; $18.8 \%$ ) e reside em meio urbano (159; 78.7\%). Relativamente ao estado civil dos participantes, $56.4 \%$ consideram-se solteiros, $24.8 \%$ casados, $11.4 \%$ encontram-se em união de facto, $6.9 \%$ assinalam a opção divorciados e $0.5 \%$ considera-se viúvo. A maioria dos sujeitos da 
amostra é residente na Região Autónoma dos Açores (60.4\%), sendo os restantes pertencentes às seguintes regiões: Norte (16.8\%); Centro (9.9\%); Lisboa e Vale do Tejo (9.9\%); Alentejo (1.5\%); Algarve (1\%) e Madeira (0.5\%). Teve-se como critério de inclusão os participantes terem idade igual ou superior a 18 anos, independentemente de estes apresentarem uma diferença visível ou não.

\section{Instrumentos}

CARSAL/CARVAL [Center for Appearance Research Salience Scale/ Center for Appearance Research Valence Scale] - (Moss \& Rosser, 2012) é composta por 13 itens de autorresposta, numa escala tipo Likert, com uma cotação que varia entre 1 (Discordo totalmente) e 6 (Concordo totalmente). A escala apresenta uma confiabilidade interna $\alpha$ de Cronbach $=.90$ para a CARSAL e $\alpha$ de Cronbach $=.93$ para a CARVAL (Moss \& Rosser, 2012). Pontuações mais altas na CARSAL indicam maior saliência da aparência dentro do autoconceito, isto é, a aparência sendo parte do autoconceito de trabalho. Pontuações mais altas na CARVAL indicam uma avaliação emocional mais negativa em relação à aparência.

DAS-24 [Derriford Appearance Scale-short] - (Carr, Moss \& Harris, 2005; Moreira \& Canavarro, 2007) tem como constructo subjacente a ser medido, a autoconsciência da aparência, num total de 24 itens, respondidos numa escala tipo Likert, com 6 questões adicionais. Os 24 itens têm como objetivo avaliar a forma como o indivíduo se sente e comporta relativamente à característica da aparência que o incomoda, sendo cotados para os itens 3, 5, 7, 9, 12, 13, 15, 18, 19, 21, 24 através das seguintes opções de resposta: "0 = N/A (Não Aplicável); 1 = Nunca/Quase nunca; 2 = Às vezes; 3 = Frequentemente; e $4=$ Quase Sempre". Para os restantes itens, aplicam-se as seguintes opções de resposta: "1 = Nada; 2 = Ligeiramente; 3 = Moderadamente; e 4 = Extremamente". 0 estudo psicométrico da versão portuguesa (Mendes et al., 2016) apresenta boas propriedades psicométricas, $\alpha$ de Cronbach $=.91$.

ASI-R [The Appearance Schemas Inventory - Revised] - (Cash, Melnyk, \& Hrabosky, 2004; Nazaré et al., 2010), composta por dois fatores (saliência auto-avaliativa e saliência motivacional), pretende avaliar o investimento esquemático na aparência (Cash et al., 2004) através de 20 itens de autorresposta, numa escala tipo Likert, com uma cotação que varia entre 1 (Discordo fortemente) e 5 (Concordo fortemente), avaliando os esforços de um indivíduo em manter ou aumentar a sua atratividade física e gerir a sua aparência. Quanto mais elevado for este resultado, maiores são os níveis de investimento esquemático relativos à aparência. A versão portuguesa do instrumento (Nazaré et al., 2010) apresenta características psicométricas idênticas à versão original $\alpha$ de Cronbach $=.89$ ).

PANAS [Positive and Negative Affect Schedule] - (Galinha \& Pais-Ribeiro, 2005; Watson, Clark, \& Tellegen, 1988) avalia estados emocionais específicos, composta por 20 emoções organizadas em duas subescalas: afetos positivos $\alpha$ de Cronbach =.86) e afetos negativos $\alpha$ de Cronbach $=.89$ ), com uma correlação entre subescalas perto de zero $(r=-.10)$. As respostas variam entre "1- nada ou muito ligeiramente, 2 - um pouco, 3 - moderadamente, 4 - bastante, e 5 - extremamente”.

\section{Procedimento}

O instrumento CARSAL/CARVAL foi traduzido e adaptado de acordo com as recomendações para a tradução de instrumentos aplicados em investigação, respeitando a equivalência da tradução do item, a equivalência operacional, a equivalência da escala e a equivalência métrica. Através da versão original CARSAL/CARVAL, dois tradutores bilingues, mestres em Psicologia Clínica e da Saúde, traduziram os itens de Inglês para Português, tendo-se aplicado a primeira tradução a 30 indivíduos, no intuito de testar as questões do instrumento (Leeuw, Hox, \& Dillman, 2008). Posteriormente, um doutorando em Psicologia (bilingue) procedeu à retradução cega dos itens para a língua inglesa, tendo-se remetido posteriormente os itens ao autor original da escala, comparando-se ambas as versões. Após unanimidade na verificação da escala entre todos os envolventes no processo de tradução, retradução e versão final da CARSAL/CARVAL para português, prosseguiu-se com a elaboração de um questionário sociodemográfico, disponibilizado na internet (Google Forms) com escalas, algumas adicionais (CARSAL/CARVAL, DAS-24, ASI-R e PANAS).

Por fim, procedeu-se à divulgação do estudo na população portuguesa, através da disponibilização de um link nas redes sociais (Facebook e Linkedin) e por correio eletrónico (efeito "bola de neve"). Além das informações referidas, o estudo disponibilizado on-line, iniciava-se com o termo de consentimento informado e esclarecido e respetivo objetivo do estudo, permitindo ao participante a total liberdade de responder às questões que se seguiam.

\section{Análise estatística}

Recorreu-se ao software SPSS24 do sistema operativo macOS e AMOS21 do sistema operativo Windows, para a realização e procedimentos estatísticos. Procedeu-se a uma análise fatorial exploratória (AFE) e verificaram-se os alpha de Cronbach $(\alpha)$ no intuito de avaliar a correlação entre os itens de forma 
a aferir a medição do constructo teórico. Posteriormente, analisou-se o coeficiente de correlação de Pearson (r) para quantificar a intensidade e a direção da associação entre as dimensões dos instrumentos CARSAL/CARVAL, DAS-24, ASI-R e PANAS.

Prosseguiu-se posteriormente à análise fatorial confirmatória (AFC), com a estimação de máxima verosimilhança, avaliando a qualidade de ajustamento global do modelo fatorial pelo teste Qui-quadrado de Ajustamento $\left(X^{2}\right)$, considerando $X^{2} / g$.l. inferior a 5 como modelo aceitável. De acordo com os índices Comparative Fit Index (CFI), Goodness of Fit Index (GFI), Root Mean Square Error of Approximation (RMSEA), considerou-se um bom ajustamento quando os índices eram próximos do valor de 1 e o RMSEA apresentasse valores de referência inferiores a .05 (Marôco, 2014). A fiabilidade compósita (FC) e a variância extraída média (VEM) foram avaliadas através das fórmulas (Marôco, 2014):

$$
\widehat{F C}=\frac{\left(\sum_{i t=1}^{5} \lambda\right)^{2}}{\left(\sum_{i t=1}^{5} \lambda\right)^{2}+\sum_{i t=1}^{5} \varepsilon} \quad \widehat{V E M}=\frac{\sum_{i t=1}^{5} \lambda^{2}}{\sum_{i t=1}^{5} \lambda^{2}+\sum_{i t=1}^{5} \varepsilon}
$$

Utilizou-se os testes $t$-Student e ANOVA, de forma a verificar diferenças estatísticas entre as variáveis, tendo em consideração o tamanho do efeito (TDE) (Espírito Santo \& Daniel, 2017). A regressão linear múltipla permitiu analisar relações entre as variáveis e avaliar possíveis variáveis preditores. A pontuação total da versão portuguesa original da CARSAL/CARVAL foi utilizada como variável dependente e cada item como variável independente.

\section{RESULTADOS}

\section{Fiabilidade}

A versão original da CARSAL/CARVAL composta por 13 itens, apresenta um índice de consistência interna de $\alpha=.90$ para a dimensão Saliência (5 itens) e $\alpha=.93$ para a dimensão Valência (8 itens). A versão portuguesa da CARSAL/CARVAL apresenta índice de consistência interna moderada para a dimensão Saliência $\alpha=.77$ ( 5 itens) e elevada para a dimensão Valência, $\alpha=.93$ (8 itens).

Ao verificar a intensidade da associação entre CARSAL/CARVAL, DAS-24, dimensões da ASI-R e PANAS, a Tabela 1 apresenta associação muito forte entre a CARVAL e a DAS-24. Verifica-se uma correlação moderada entre a CARSAL e as dimensões da ASI-R e uma intensidade moderada entre a DAS24, Saliência Auto-Avaliativa e Afeto Negativo.

Tabela 1. Correlação entre a CARSAL/CARVAL, Dimensões do ASI-R, DAS-24, Afeto Positivo e Afeto Negativo

\begin{tabular}{lccccccc}
\hline & $\mathbf{1}$ & $\mathbf{2}$ & $\mathbf{3}$ & $\mathbf{4}$ & $\mathbf{5}$ & $\mathbf{6}$ & $\mathbf{7}$ \\
\hline 1. CARSAL & - & .109 & $.260^{* *}$ & $.569^{* *}$ & $.503^{* *}$ & .077 & $.332^{* *}$ \\
2. CARVAL & & -- & $.761^{* *}$ & $.520^{* *}$ & .075 & $-.465^{* *}$ & $.398^{* *}$ \\
3. DAS-24 & & & -- & $.638^{* *}$ & $.154^{*}$ & $-.422^{* *}$ & $.574^{* *}$ \\
4. Saliência: auto-avaliativa & & & & -- & $.561^{* *}$ & $-.231^{* *}$ & $.506^{* *}$ \\
5. Saliência: motivacional & & & & & -- & .120 & $.184^{* *}$ \\
6. Afeto Positivo & & & & & & -- & .036 \\
7. Afeto Negativo & & & & & & -- \\
\hline$M$ & & & & & & & \\
$D P$ & 21.88 & 26.43 & 41.48 & 41.03 & 27.20 & 29.21 & 21.56 \\
\hline$*$
\end{tabular}

${ }^{*} p<.05 .{ }^{* *} p<.01 .{ }^{* * *} p<.001$.

\section{Análise Fatorial Exploratória}

Procedeu-se ao método de análise de componentes principais, com rotação Varimax, tendo-se verificado os itens de cada dimensão. A medida de Kaiser-Meyer-Olkin verificou homogeneidade das variáveis, o que se revela adequado à amostra em análise (KMO = .89). Através do teste de esfericidade de Bartlett $\left(X^{2}(78)=1807.29 ; p=<.001\right)$ verifica-se que se as variáveis estão suficientemente correlacionadas a ponto de ser apropriado realizar uma análise fatorial exploratória. Na Tabela 2, constata-se que as correlações item-total variam entre .53 e .90, verificando-se correlações moderadas a elevadas na maioria dos itens. A estrutura da AFE sugere que a estrutura original se adequa aos dados portugueses, com exceção do primeiro item $(\mathrm{r}=.53)$. 
Tabela 2. Comparação das Correlações do Item-Toral da CARSAL/CARVAL e Item-Total da CARSAL/CARVAL Versão Original

\begin{tabular}{|c|c|c|c|}
\hline Item & Descrição do Item & $\begin{array}{l}\text { Correlação: Item- } \\
\text { Total }(r)\end{array}$ & $\begin{array}{c}\text { Correlação: Item- } \\
\text { Total Versão } \\
\text { Original }(r) \\
\end{array}$ \\
\hline 1 & $\begin{array}{l}\text { Para mim, a minha aparência é uma parte importante do } \\
\text { que eu sou. }\end{array}$ & .527 & .724 \\
\hline 2 & $\begin{array}{l}\text { Muitas vezes tenho consciência da maneira como sou } \\
\text { olhado pelas outras pessoas. }\end{array}$ & .767 & .752 \\
\hline 3 & $\begin{array}{l}\text { Na maioria das situações, dou por mim a ter consciência da } \\
\text { maneira como a minha face e corpo parecem. }\end{array}$ & .846 & .810 \\
\hline 4 & $\begin{array}{l}\text { Penso muitas vezes no impacto que a aparência do meu } \\
\text { corpo e face transmitem. }\end{array}$ & .820 & .759 \\
\hline 5 & Geralmente estou consciente da minha aparência. & .614 & .773 \\
\hline 6 & Estou satisfeito(a) com a minha aparência física. * & .870 & .802 \\
\hline 7 & Não gosto da minha aparência. & .807 & .737 \\
\hline 8 & A minha aparência faz-me sentir bem comigo mesmo(a). * & .881 & .775 \\
\hline 9 & A minha aparência é pouco atraente. & .755 & .716 \\
\hline 10 & $\begin{array}{l}0 \text { meu corpo e a minha face parecem tão bem como eu } \\
\text { gostaria. } *\end{array}$ & .763 & .760 \\
\hline 11 & Sinto-me mal com o meu corpo e a minha aparência. & .784 & .714 \\
\hline 12 & Gosto da minha aparência. * & .902 & .844 \\
\hline 13 & A minha aparência faz-me sentir atraente. ${ }^{*}$ & .894 & .758 \\
\hline
\end{tabular}

Nota $*$ itens invertidos

\section{Análise Fatorial Confirmatória}

A estrutura das dimensões CARSAL/CARVAL apresenta índices de qualidade de ajustamento considerados sofríveis $\left(X^{2} / \mathrm{df}=4.78\right.$; $\mathrm{CFI}=.86$; GFI = .79; $\mathrm{TLI}=.83$; $\mathrm{RMSEA}=.137 ; p$ [rmsea $\left.\leq .05\right]=$ $<.001)$. A regressão e as variâncias de todos os itens são significativas $(p=<.001)$. Tendo-se verificado um ajustamento considerado sofrível, procedeu-se à refinação do modelo pelas maiores covariâncias apresentadas pelo índice de modificação obtido (Figura 1). 0 modelo apresenta-se com um ajustamento considerado bom quando a reespeficicação do modelo pela correlação dos erros de medida nos itens 7, 9 e $11\left(X^{2} / \mathrm{df}=2.99 ; \mathrm{GFI}=.88 ; \mathrm{CFI}=.93 ; \mathrm{TLI}=.91 ; \mathrm{RMSEA}=.100 ; p[\mathrm{rmsea} \leq .05]=<.001\right)$.

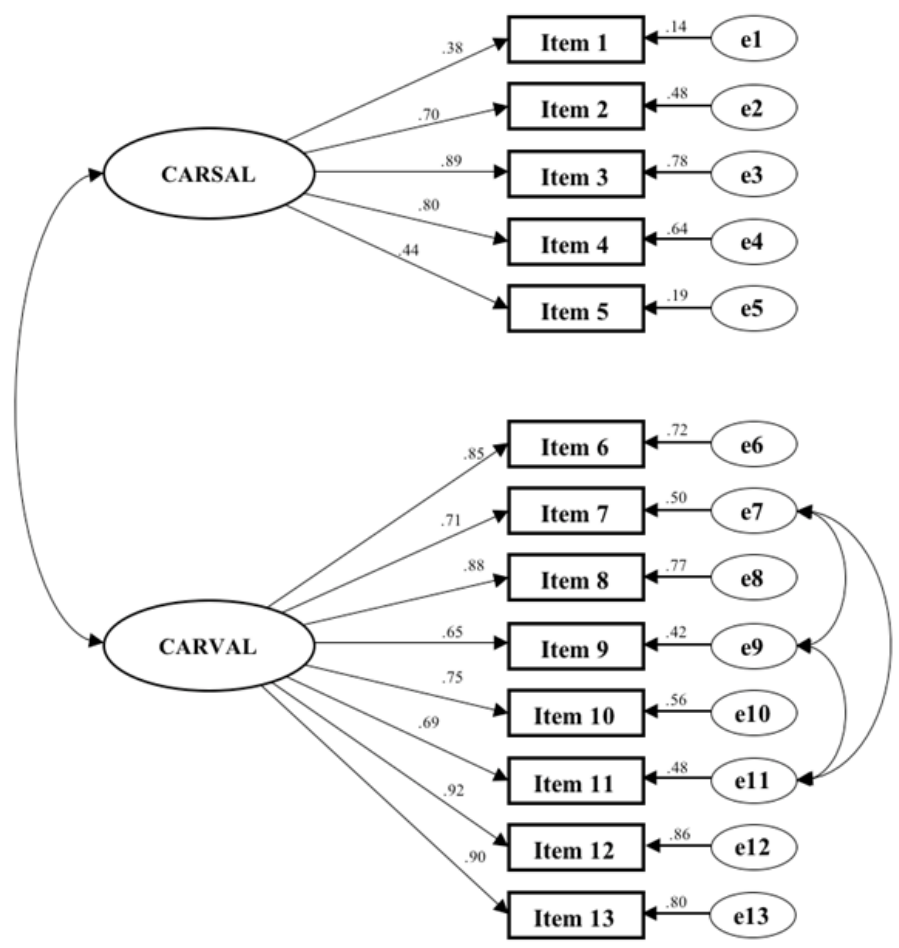

Figura 1. Modelo de Análise Fatorial Confirmatória da CARSAL/CARVAL (X2/df=2.997; GFI=.93; $\mathrm{GFI}=.88 ; \mathrm{TLI}=.91 ; \mathrm{RMSEA}=.100 ; \mathrm{p}=<.001 ; \mathrm{n}=202$ ). 
Pelos pesos fatoriais estandardizados, calculou-se a fiabilidade compósita, que se revelou apropriada quer para a CARSAL $(\widehat{F C}=.79)$ quer para a CARVAL $(\widehat{F C}=.93)$. A variância extraída média, apresenta um valor baixo para a CARSAL $(\widehat{V E M}=.45)$ e indicador de validade convergente adequada para a CARVAL $(\widehat{V E M}=.74)$.

\section{Análise de Regressões}

Considerando a pontuação total da escala CARSAL/CARVAL como variável dependente e os itens que a compõem como variáveis independentes, verificou-se um modelo perfeito para a amostra em estudo $\left(R^{2}=1\right)$, com correlações que variam entre .59 e .89. A regressão linear múltipla permitiu identificar as variáveis DAS-24 CARSAL/CARVAL como preditores significativos. 0 modelo significativo, prevê que a DAS-24 represente $60.8 \%$ da variabilidade na variável dependente; ambos os preditores providenciam contribuições significativas independentes, CARSAL $(\beta=.18$, t $(199)=4.02 ; p=<.001) \mathrm{e}$ CARVAL $(\beta=.74, \mathrm{t}(199)=16.69 ; p=<.001)$.

\section{Comparação de Médias}

A realização de teste $t$-Student permitiu verificar diferenças significativas entre a variável sexo e as variáveis DAS-24 e Saliência Auto-Avaliativa. O sexo feminino apresenta uma média maior sobre as preocupações em relação à aparência $(\mathrm{M}=1.78$, $\mathrm{DP}=.56)$ em comparação com o sexo masculino $(\mathrm{M}=$ $1.49, \mathrm{DP}=.55)$ e valorizam mais a gestão da aparência $(\mathrm{M}=3.48, \mathrm{DP}=.73)$ em comparação com o sexo masculino $(\mathrm{M}=3.13$, $\mathrm{DP}=3.13)$. De acordo com o teste $t$-student, as diferenças observadas entre os dois grupos, são estatisticamente significativas ( $\mathrm{t}(200)=-2.833 ; p=.005$; TDE considerado médio, $d$ de Cohen $=.51$; IC 95\% [.15; .86]) quer para a DAS-24 quer para a Saliência Auto-Avaliativa (t $(200)=-2.555 ; p=$ .011 ; TDE considerado pequeno, $d$ de Cohen $=.46$; IC 95\% $[.10 ; .81]$ ). Não se verificaram diferenças significativas entre as restantes variáveis (Saliência Motivacional, CARSAL/CARVAL e PANAS).

Na realização de uma ANOVA, não se verificaram diferenças estatisticamente significativas entre as restantes variáveis sociodemográficas e respetivas dimensões da CARSAL/CARVAL (saliência/valência), DAS-24 (autoconsciência da aparência), ASI-R (saliência auto-avaliativa e saliência motivacional) e PANAS (afeto positivo e afeto negativo).

\section{DISCUSSÃO}

Tomando por base a escala original da CARSAL/CARVAL, pretende-se com este estudo, contribuir para a adaptação de um instrumento que avalie dois aspetos do auto-esquema da aparência (valência e saliência) para a população portuguesa.

Os resultados apresentaram um índice de consistência interna moderada para a CARSAL e uma elevada consistência interna para a CARVAL. O estudo original de Moss e Rosser (2012), a validação da CARSAL e CARVAL, iniciou-se com 22 questões, tendo-se procedido à eliminação de 9 itens, por apresentarem correlações muito baixas. Comparativamente à fiabilidade da CARSAL, esta apresentou um valor inferior à escala original ( $\alpha=.77$ vs $\alpha=.93$ ), considerado um valor adequado (Widaman et al., 2011), tendo a CARVAL apresentado uma consistência interna igual à escala original $(\alpha=.93)$. Estas diferenças podem dever-se a efeitos socioculturais, que poderão ser responsáveis pela influência na internalização de ideias relativas à aparência (Clark \& Tiggemann, 2008). Moss e colaboradores (2014) referem que a aplicação da CARSAL/CARVAL em indivíduos com diferenças visíveis, apresenta uma fiabilidade mais baixa, podendo esta redução de fiabilidade estar relacionada com a sua aplicação em contexto clínico, no entanto reforçamos que amostra deste estudo considera-se não clínica, sugerindo-se futuros estudos numa amostra (sub)clínica portuguesa.

A análise fatorial exploratória verificou a homogeneidade das variáveis (KMO > .80), considerada boa (Marôco, 2018), apresentando correlações moderadas a elevadas para a CARSAL/CARVAL ( $\mathrm{r}>.50 ; \mathrm{r}$ <.91). Tendo em consideração as regras do eigenvalue superior a 1 e o Scree plot, a avaliação do modelo fatorial permitiu determinar dois fatores (Marôco, 2018).

A análise fatorial confirmatória, para a testagem do modelo de equação estrutural apresentou-se inicialmente sofrível, no entanto a reespecificação do modelo pelos erros, permitiu apresentar índices de qualidade de ajustamento considerados bons $\left(X^{2} / \mathrm{df}=2.99 ; \mathrm{GFI}=.88 ; \mathrm{CFI}=.93\right.$; TLI=.91; RMSEA=.100; $p[$ rmsea $\leq .05]=<.001$ ) (Marôco, 2014).

A fiabilidade compósita da CARSAL ( FC = .79) e CARVAL $(F C=.93)$ é indicadora de uma fiabilidade de constructo e validade convergente apropriada (c.f. Marôco, 2014, pp.183-184). Na verificação de possíveis relações entre as dimensões dos instrumentos, constatou-se que a DAS-24 apresentou uma correlação muito forte e significativa $(|r| \geq .75$, Marôco, 2018) com a CARVAL. Esta associação pode dever-se ao facto de os participantes em estudo apresentarem valores acima da média DAS-24, que indicam sentimentos de desconforto em relação à sua aparência (Mendes et al., 2016; Mendes \& Pereira, 
2018) e valores acima da média na CARVAL, que indicam uma avaliação mais negativa da aparência (Moss \& Rosser, 2012).

Estes resultados vão ao encontro dos resultados da versão original, incluindo também a CARSAL/CARVAL como preditora da autoconsciência da aparência e a existência de diferenças significativas entre a variável género e autoconsciência da aparência (Moss \& Rosser, 2012). Salienta-se que não se verificaram outras diferenças significativas entre as restantes variáveis.

Partindo de que o presente estudo prévio, de adaptação e análise psicométrica da CARSAL/CARVAL para a população portuguesa foi aplicado a uma amostra não clínica, verificou-se que os resultados vão ao encontro do estudo original (Moss \& Rosser, 2012). Apesar das limitações existentes neste estudo, por exemplo, o número da amostra ser inferior ao do estudo original, a versão portuguesa da escala CARSAL/CARVAL apresenta boa homogeneidade das variáveis, em que as retenções dos dois fatores explicam cerca de $66.40 \%$ da variabilidade total. 0 instrumento está adaptado para o estudo dos auto-esquemas da aparência (saliência e valência) na população Portuguesa.

\section{CONCLUSÃO}

A escala CARSAL/CARVAL desenvolvida por Moss e Rosser (2012) é uma medida que avalia dois aspetos do auto-esquema da aparência; com aplicação em contexto geral e clínico. A importância deste instrumento evidencia-se pela emergência da Psicologia da Aparência em Portugal, uma vez que a comunidade científica tem demonstrado um incrementado interesse em melhor compreender a influência da aparência no quotidiano dos indivíduos, considerando-se um instrumento importante na compreensão de distintos componentes do autoconceito relacionados com a aparência de um indivíduo.

\section{Agradecimentos}

Ao Professor Doutor Timothy Moss, pela autorização e avaliação da tradução da CARSAL/CARVAL.

\section{REFERÊNCIAS}

Brill, S., Clarke, A., Veale, D., \& Butler, P. (2006). Psychological management and body image issues in facial transplantation. Body Image, 3(1), 1-15. https://doi.org/10.1016/j.bodyim.2005.12.002

Carr, T., Moss, T., \& Harris, D. (2005). The DAS24: a short form of the Derriford Appearance Scale DAS59 to measure individual responses to living with problems of appearance. British Journal of Health Psychology, 10(2), 285-298. https://doi.org/10.1348/135910705X27613

Cash, T. (2006). The Influence of Sociocultural Factors on Body Image: Searching for Constructs. Clinical Psychology: Science and Practice, 12(4), 438-442. https://doi.org/10.1093/clipsy.bpi055

Cash, T., \& Pruzinsky, T. (2004). Body image: a handbook of theory, research, and clinical practice. New York, NY: Guilford Press.

Cash, T., \& Smolak, L. (2012). Body Image: A handbook of Science, Practice, and Prevention (2sd ed.). New York: The Guilford Press.

Cash, T., Melnyk, S., \& Hrabosky, J.(2004). The assessment of body image investment: an extensive revision of the appearance schemas inventory. The International Journal of Eating Disorders, 35(3), 305-316. https://doi.org/10.1002/eat.10264

Clark, L., \& Tiggemann, M. (2008). Sociocultural and individual psychological predictors of body image in young girls: A prospective study. Developmental Psychology, 44(4), 1124-1134. https://doi.org/10.1037/0012-1649.44.4.1124

Clarke, A., Thompson, A., Jenkinson, E., Rumsey, N., \& Newell, R. (2014). CBT for Appearance Anxiety: Psychosocial Interventions for Anxiety due to Visible Difference. United Kingdom: Wiley Blackwell.

Deno, M., Tashiro, M., Miyashita, M., Asakage, T., Takahashi, K., Saito, K., ... Ichikawa, Y. (2012). The mediating effects of social support and self-efficacy on the relationship between social distress and emotional distress in head and neck cancer outpatients with facial disfigurement. Psycho-Oncology, 21(2), 144-152. https://doi.org/10.1002/pon.1877

Espírito Santo, H., \& Daniel, F. (2017). Calcular e apresentar tamanhos do efeito em trabalhos científicos (2): Guia para reportar a força das relações. Revista Portuguesa De Investigação Comportamental E Social, 3(1), 53-64. https://doi.org/10.7342/ismt.rpics.2017.3.1.48

Fuller-Tyszkiewicz, M., Chhouk, J., McCann, L.-A., Urbina, G., Vuo, H., Krug, I., ... Richardson, B. (2019). Appearance comparison and other appearance-related influences on body dissatisfaction in everyday life. Body Image, 28, 101-109. https://doi.org/10.1016/j.bodyim.2019.01.002

Galinha, I. C., \& Pais-Ribeiro, J. L. (2005). Contribuição para o estudo da versão portuguesa da Positive and Negative Affect Schedule (PANAS): II - Estudo psicométrico. Análise Psicológica, 23(2), 219-227. Retirado de http://www.scielo.mec.pt/pdf/aps/v23n2/v23n2a12.pdf 
Hockenberry, M. J., \& Wilson, D. (2011). Wong Enfermagem da Criança e do Adolescente (9th ed, Vol. 1). Loures: Lusociência.

Lansdown, R., Rumsey, N., Bradbury, E., Carr, T., \& Partridge, J. (1997). Visibly different: coping with disfigurement. Oxford ; Boston: Butterworth-Heinemann.

Leeuw, E., Hox, J., \& Dillman, D. (2008). International handbook of survey methodology. New York; London: Lawrence Erlbaum Associates.

Lonergan, A. R., Bussey, K., Mond, J., Brown, O., Griffiths, S., Murray, S., \& Mitchison, D. (2019). Me, my selfie, and I. The relationship between editing and posting selfies and body dissatisfaction in men and women. Body Image, 28, 39-43. https://doi.org/10.1016/j.bodyim.2018.12.001

Marôco, J. (2014). Análise de Equações Estruturais: fundamentos teóricos, software \& aplicações. Lisboa: ReportNumber.

Marôco, J. (2018). Análise Estatística com o SPSS Statistics (7th ed.). Lisboa: Report Number.

Mendes, J. (2017). Fatores Determinantes no Ajustamento Psicológico ao Desfiguramento Facial Adquirido: a importância da autorregulação, representação cognitiva, emoções e autoconceito (tese de doutoramento). Lisboa: ISPA. https://doi.org/10.13140/RG.2.2.11192.44806

Mendes, J., \& Pereira, V. (2018). Versão Portuguesa Reduzida da Escala de Avaliação da Aparência de Derriford (DAS-14): Análise fatorial exploratória e confirmatória. Revista Portuguesa de Investigação Comportamental e Social, 4(2), 25-32. https://doi.org/10.31211/rpics.2018.4.2.79

Mendes, J., Figueiras, M. J., Moreira, H., \& Moss, T. (2016). Análise fatorial da versão portuguesa da Escala de Avaliação da Aparência de Derriford (DAS-24). Psychology, Community \& Health, 5(1), 31-43. https://doi.org/10.5964/pch.v5i1.128

Moreira, H., \& Canavarro, M. C. (2007). The Portuguese version of the Derriford Appearance Scale - 24. Unpublished manuscript, Departamento de Psicologia, Universidade de Coimbra, Portugal.

Moss, T., \& Rosser, B. A. (2012). The Moderated Relationship of Appearance Valence on Appearance Self Consciousness: Development and Testing of New Measures of Appearance Schema Components. PLoS ONE, 7(11), e50605. https://doi.org/10.1371/journal.pone.0050605

Moss, T., Lawson, V., White, P., \& The Appearance Research Collaboration. (2014). Salience and Valence of Appearance in a Population with a Visible Difference of Appearance: Direct and Moderated Relationships with Self-Consciousness, Anxiety and Depression. PLoS ONE, 9(2), e88435. https://doi.org/10.1371/journal.pone.0088435

Nazaré, B., Moreira, H., \& Canavarro, M. C. (2010). Uma perspectiva cognitivo-comportamental sobre o investimento esquemático na aparência: Estudos psicométricos do Inventário de Esquemas sobre a Aparência (ASI-R). Laboratório de Psicologia, (8), 21-36. Retirado de https://estudogeral.sib.uc.pt/bitstream/10316/14292/1/Vers\%C3\%A3o\%20final.pdf

Pitron, V., \& De Vignemont, F. (2017). Beyond differences between the body schema and the body image: insights from body hallucinations. Consciousness and Cognition, 53, 115-121. https://doi.org/10.1016/j.concog.2017.06.006

Pitron, V., Alsmith, A., \& De Vignemont, F. (2018). How do the body schema and the body image interact? Consciousness and Cognition, 65, 352-358. https://doi.org/10.1016/j.concog.2018.08.007

Smith, G., McCarthy, D., \& Anderson, K. (2000). On the sins of short-form development. Psychological Assessment, 12(1), 102-111. https://doi.org/10.1037//1040-3590.12.1.102

Tambs, K., \& Røysamb, E. (2014). Selection of questions to short-form versions of original psychometric instruments in MoBa. Norsk Epidemiologi, 24(1-2). https://doi.org/10.5324/nje.v24i1-2.1822

Watson, D., Clark, L., \& Tellegen, A. (1988). Development and validation of brief measures of positive and negative affect: the PANAS scales. Journal of Personality and Social Psychology, 54, 1063-1070. Retirado de http://www.cnbc.pt/jpmatos/28.Watson.pdf

Widaman, K., Little, T., Preacher, K., \& Sawalani, G. (2011). On creating and using short forms of scales in secondary research. In K. H. Trzesniewski, M. B. Donnellan, \& R. E. Lucas (Eds.), Secondary data analysis: An introduction for psychologists (pp. 39-61). Washington, DC US: American Psychological Association.

Historial do artigo

Recebido $\quad 10 / 18$

Aceite $\quad 07 / 19$

Publicado $\quad 08 / 19$ 